\title{
Neogenin and RGMa Control Neural Tube Closure and Neuroepithelial Morphology by Regulating Cell Polarity
}

\author{
Nigel Kee, ${ }^{1}$ Nicole Wilson, ${ }^{2}$ Melissa De Vries, ${ }^{1}$ DanaKai Bradford, ${ }^{1}$ Brian Key, ${ }^{2}$ and Helen M. Cooper ${ }^{1,2}$ \\ ${ }^{1}$ Queensland Brain Institute and ${ }^{2}$ School of Biomedical Sciences, The University of Queensland, Queensland, Brisbane 4072, Australia
}

In humans, neural tube closure defects occur in 1:1000 pregnancies. The design of new strategies for the prevention of such common defects would benefit from an improved understanding of the molecular events underlying neurulation. Neural fold elevation is a key morphological process that acts during neurulation to drive neural tube closure. However, to date, the molecular pathways underpinning neural fold elevation have not been elucidated. Here, we use morpholino knock-down technology to demonstrate that Repulsive Guidance Molecule (RGMa)-Neogenin interactions are essential for effective neural fold elevation during Xenopus neurulation and that loss of these molecules results in disrupted neural tube closure. We demonstrate that Neogenin and RGMa are required for establishing the morphology of deep layer cells in the neural plate throughout neurulation. We also show that loss of Neogenin severely disrupts the microtubule network within the deep layer cells suggesting that Neogenin-dependent microtubule organization within the deep cells is essential for radial intercalation with the overlying superficial cell layer, thereby driving neural fold elevation. In addition, we show that sustained Neogenin activity is also necessary for the establishment of the apicobasally polarized pseudostratified neuroepithelium of the neural tube. Therefore, our study identifies a novel signaling pathway essential for radial intercalation and epithelialization during neural fold elevation and neural tube morphogenesis.

Key words: Neogenin; RGMa; cell polarity; neuroepithelium; neural tube; neurulation

\section{Introduction}

The earliest neural structure in the vertebrate is the neural tube comprising a layer of pseudostratified epithelium surrounding a central lumen. The neural tube derives from a flat sheet of neuroectoderm (neural plate) arising on the dorsal surface of the blastoderm at the end of gastrulation (Lowery and Sive, 2004; Copp, 2005; Wallingford, 2005). At the onset of neurulation, the lateral regions of the neural plate elevate producing the neural folds (NFs). As the folds continue to elevate and migrate toward the dorsal midline they bend around hinge-points forming the neural groove. Finally, the dorsal tips of the NFs fuse over the midline, generating a closed neural tube. Overall, progression through neurulation requires tight spatiotemporal regulation of cell polarity, shape, and motility as well as cell-cell contact.

Much of our current understanding of neural tube formation comes from the study of Xenopus neurulation. Throughout posterior (hindbrain, spinal cord) neurulation in the Xenopus embryo, convergent extension (the process by which intercalation of the neuroectodermal and underlying mesenchymal cells narrows

Received Sept. 5, 2008; revised 0ct. 7, 2008; accepted 0ct. 15, 2008.

This work was supported by the National Health and Medical Research Council of Australia. N.W., M.D.V., and D.B. were supported by Australian Postgraduate Awards. The E7 and Xen 1 antibodies, developed by M. Klymkowsky and Ruiz i Altaba, respectively, were obtained from the Developmental Studies Hybridoma Bank developed under the auspices of the National Institute of Child Health and Human Development and maintained by The University of lowa (lowa City, IA). We thank Rowan Tweedale for her critical reading of this manuscript and Grace J. Shin Lah for assistance with the generation of embryos.

Correspondence should be addressed to Dr. Helen M. Cooper, The Queensland Brain Institute, The University of Queensland, Queensland, Brisbane 4072, Australia. E-mail: h.cooper@uq.edu.au.

DOI:10.1523/JNEUROSCI.4265-08.2008

Copyright $\odot 2008$ Society for Neuroscience $\quad 0270-6474 / 08 / 2812643-11 \$ 15.00 / 0$ the embryo along the mediolateral axis while lengthening the anteroposterior axis) reduces the distance between the NFs allowing apposition at the midline (Keller et al., 1992; Wallingford and Harland, 2002). Failure of this process results in posterior neural tube closure defects. However, convergent extension contributes little to anterior neural tube closure (Keller et al., 1992; Copp, 2005; Wallingford, 2005). Instead, NF elevation and neural plate bending are the primary forces driving anterior neurulation. Neural plate bending is generated by the constriction of actin filaments under the apical surface of the neuroectodermal cells (apical constriction) leading to the formation of hingepoints on either side of the dorsal midline (Haigo et al., 2003; Lee et al., 2007). Inability to undergo apical constriction results in failure of anterior neural tube closure (anencephaly) (Hildebrand and Soriano, 1999; Haigo et al., 2003). To date, the molecular mechanisms promoting NF elevation have not been identified. Here we demonstrate that NF elevation is dependent on the axon guidance receptor, Neogenin (Vielmetter et al., 1994; Keeling et al., 1997; De Vries and Cooper, 2008).

Neogenin has been shown to act as a chemoattractive axon guidance receptor upon Netrin-1 binding, and a chemorepulsive receptor when binding Repulsive Guidance Molecule (RGMa) (Rajagopalan et al., 2004; Wilson and Key, 2006). Neogenin is also required for adhesion between multipotent progenitor cells and the adjacent epithelial layer of the developing mammary gland (Srinivasan et al., 2003). In addition, Netrin-1-Neogenin interactions promote adhesion and migration of vascular smooth muscle cells, and induce myotube formation in vitro (Kang et al., 2004; Park et al., 2004). Evidence that Neogenin is required for successful neurulation comes from our previous study in the ze- 
brafish where loss of Neogenin resulted in a severe disruption of neural tube morphology (Mawdsley et al., 2004). Here, we demonstrate that RGMa and Neogenin are essential for neural tube formation as they establish apicobasal polarity during NF elevation and also in the neuroepithelium after neural tube closure.

\section{Materials and Methods \\ In situ hybridization}

Xenopus laevis borealis embryos were obtained and reared as previously described (Connor and Key, 2002). Embryos were staged according to Nieuwkoop and Faber (1994). The use of animals as described here was approved by the Animal Ethics Committee of the University of Queensland in accordance with the guidelines stipulated by the National Health and Medical Research Council of Australia. A $3.1 \mathrm{~kb} 5^{\prime}$ Xneogla cDNA fragment (Wilson and Key, 2006) was used as the template for the synthesis of the riboprobes. It should be noted that two distinct Xneog genes (Xneog1a and Xneog1b) have been identified in the Xenopus genome (N. H. Wilson and B. Key, unpublished observations). The riboprobe used in this analysis does not discriminate between the two. The DIG RNA Labeling Kit (Roche Diagnostics) was used to synthesize the digoxigenin (DIG)labeled riboprobes. Embryos were fixed with $4 \%$ paraformaldehyde (PFA) and in situ hybridization was performed as described in the DIG Application Manual for Nonradioactive In situ Hybridization (Roche). Bound riboprobe was visualized using an anti-DIG-AP Fab fragment (Roche) followed by the BM Purple chromatogenic reaction (Roche).

\section{Morpholinos and cRNA}

The target sequences of all Xenopus morpholinos (MOs) are listed in supplemental Figure 1, available at www.jneurosci.org as supplemental material. Additional specificity controls included XplexinA and Xneuropilin1 MOs (supplemental Fig. 1, available at www.jneurosci.org as supplemental material). Zebrafish morpholinos are described by Mawdsley et al. (2004). MOs were synthesized by GeneTools. The standard control MO (ContMO) from GeneTools was used as a nonspecific control. Capped RNA (cRNA) encoding full-length Xneog1b was synthesized using the mMessage mMachine cRNA Transcription Kit (Ambion).

\section{Blastomere injections and phenotype analysis}

Xenopus. Fertilized eggs were collected and two-cell stage blastomeres injected as previously described (Connor and Key, 2002). The neurulation delay in the morphants was quantitated as follows: the number of morphants at each embryonic stage was determined when $90 \%$ of ContMO embryos had reached stage 18 (st18). The mean number of delayed embryos over a minimum of three independent experiments was then expressed as a percentage of the total number of embryos injected with that MO $( \pm$ SEM). The delay phenotype was analyzed using ANOVA followed by Bonferroni's or Dunnett's post hoc test, or by the KruskalWallis test followed by the Mann-Whitney test.

Zebrafish. Zebrafish embryos were injected with $2 \mathrm{ng}$ of zfcont or zfneogATG MO as previously described (Mawdsley et al., 2004).

\section{Immunohistochemistry}

The mouse anti- $\beta$-tubulin antibody (E7) (1:150) and the neural specific Xen 1 antibody (1:500) were obtained from the Developmental Studies Hybridoma Bank (University of Iowa, Iowa City, IA). Xenopus embryos were fixed in $80 \%$ methanol, $20 \%$ DMSO and incubated for $2 \mathrm{~h}$ in block-
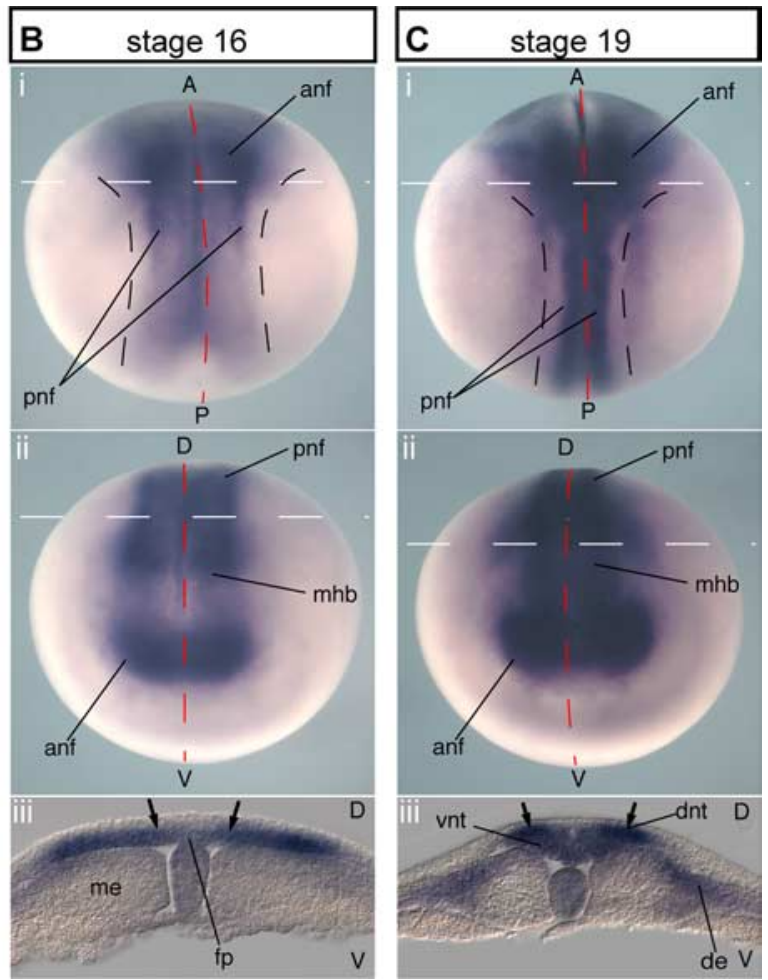

Figure 1. Xneog is expressed in the deep layer of the lateral and intermediate neural plate during neurulation. Xneog is (he deep layer of the lateral regions of the st13 posterior neural plate (pnp) (Ai, Aiii, arrows) and the anterio the , frontal view; (iii), transverse view at the level of the white dashed line in above panels. Dashed red lines indicate midline. A Anterior; bp, blastopore; de, dermamyotome; D, dorsal; dnt, dorsal neural tube; me, mesoderm; mhb, midbrain-hindbrain junction; nc, notocord; P, posterior; V, ventral; vnt, ventral neural tube.

ing solution ( $2 \%$ fetal calf serum, $2 \%$ goat serum, $0.2 \%$ Triton $\mathrm{X}-100$ in PBS), then overnight at $4^{\circ} \mathrm{C}$ with primary antibodies in blocking solution. Mouse anti-ZO-1 antibody (Zymed Laboratories) was used at 1:70. Zebrafish embryos were fixed for $12 \mathrm{~h}$ in $4 \%$ PFA at $4^{\circ} \mathrm{C}$, washed in PBS, incubated in $30 \%$ sucrose for $2 \mathrm{~h}$ and then embedded in Tissue-Tek OCT medium. Sections $(20 \mu \mathrm{m})$ were rehydrated in PBS, permeabilized in PBD (PBS containing $0.1 \%$ Tween 20 and $0.5 \%$ Triton X-100), and blocked for $1 \mathrm{~h}$ with $20 \%$ goat serum and $1 \%$ BSA in PBD. Sections were incubated with primary antibodies in blocking solution overnight at $4^{\circ} \mathrm{C}$. Bound antibody was visualized with goat anti-mouse IgG-AlexaFluor 488 (1:1000; Invitrogen). DiI (1, 1'-dioctadecyl-3, 3, 3', 3'tetramethylindocarbocyanine perchlorate; Invitrogen) was used to label the plasma membrane of neuroepithelial cells in zebrafish embryos. Frozen sections were incubated in $100 \mu \mathrm{M}$ DiI for $2 \mathrm{~h}$ and then washed extensively with PBD after application of the secondary antibody. Images were acquired on an Olympus IX81 microscope using AnalySIS software or on a Zeiss Axioplan 2 microscope using Axiovision software. Higher resolution images were obtained using a Leica LSM laser scanning confocal microscope or Zeiss LSM 510 Meta confocal microscope.

\section{Results}

\section{Loss of Xneog results in failure of neural tube closure}

We initially determined the expression pattern of Xenopus Neogenin (Xneog) mRNA from st11 through to st19 of development in the Xenopus embryo by in situ hybridization. The Xenopus neural plate comprises a superficial layer of flat, neuroectodermal cells and a deep layer of densely packed mesenchymal-like cells which have lengthened in the apicobasal dimension (Keller, 2002; Wallingford, 2005). Intense Xneog expression was first detected at the beginning of neurulation (st13), with the highest expression localized to the deep layer of the lateral neural plate at the level of 
A
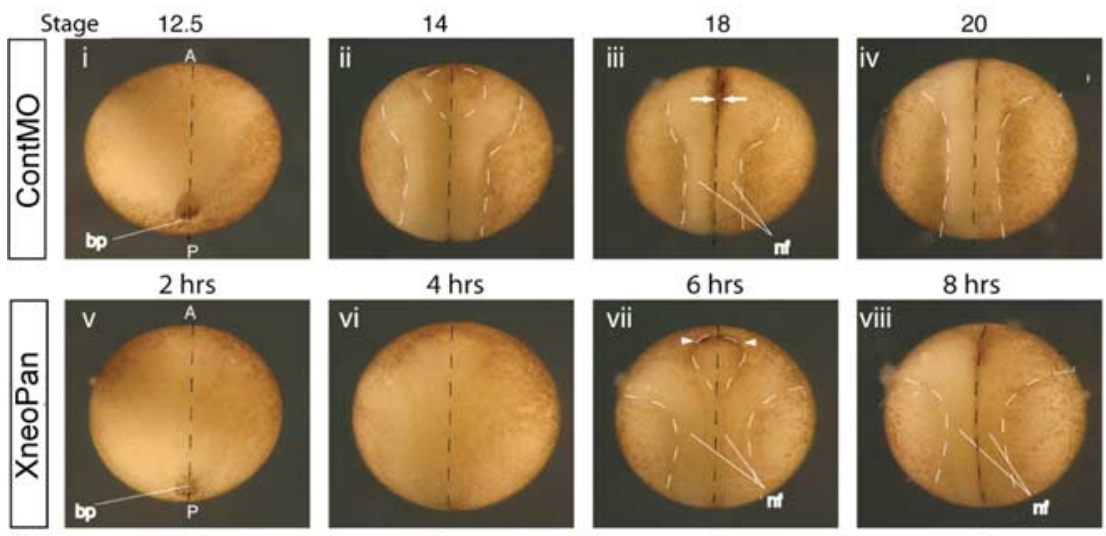

NF Stage

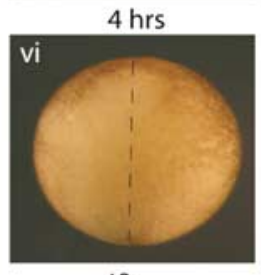

13

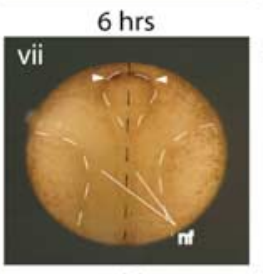

16

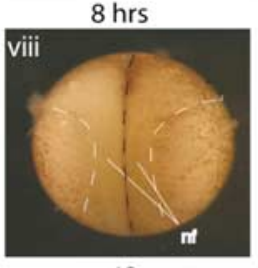

19
B

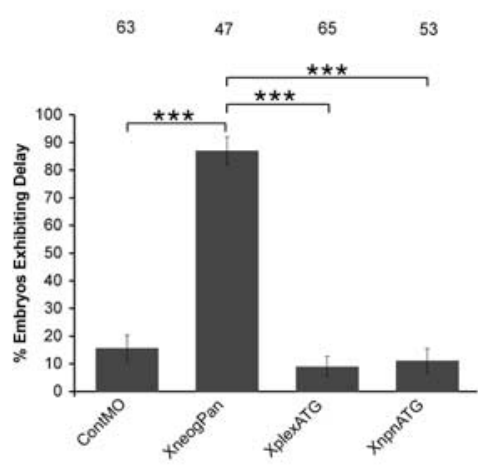

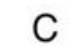

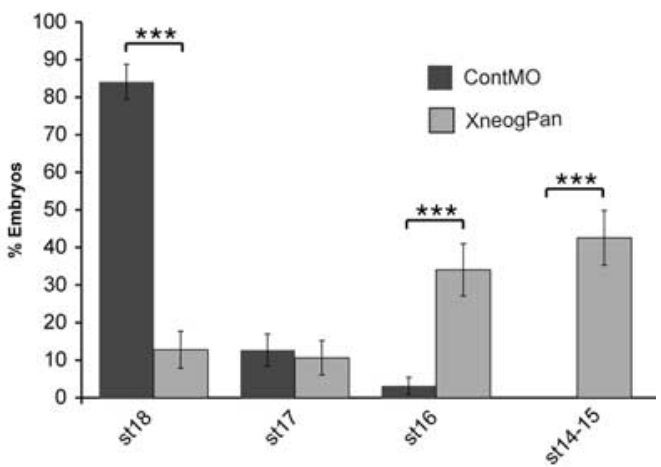

E
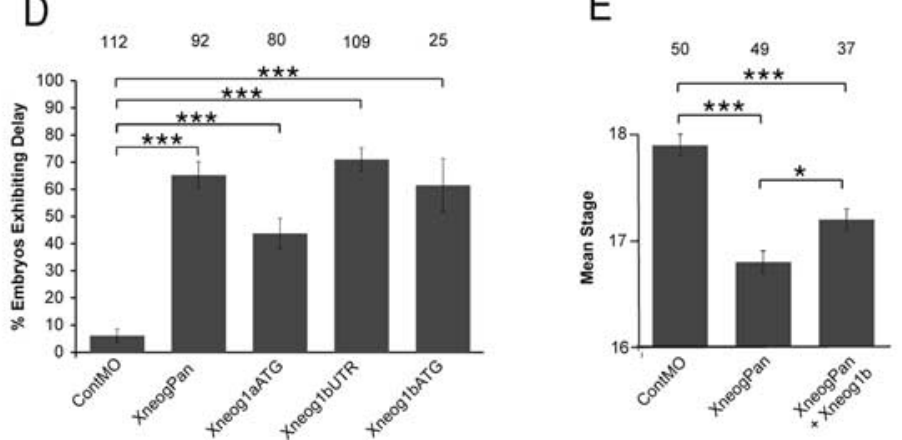

Figure 2. Loss of Xneog results in neural tube closure defects. $\boldsymbol{A}$, Injection of $16 \mathrm{ng}$ of XneogPan M0 but not ContM0 results in delayed neural tube closure (dorsal view). Hours indicate time since embryos were at st11. Dashed white lines indicate the elevating NFs. Dashed black lines indicate midline. Aiii, Arrows: NF apposition. Avii, Arrowheads: NFs have failed to reach apposition. B, Quantification of the number of embryos exhibiting a neurulation delay when injected with ContM0, XneogPan M0, XplexATG MO or XnpnATG MO. C, Percentage of XneogPan-injected embryos at each stage of neurulation when $90 \%$ of ContM0injected embryos have reached st 18. D. Percentage of embryos exhibiting a neurulation delay when injected with $5^{\prime}$ UTR and ATG MOs specific for Xneog1a and Xneog1b. $\boldsymbol{E}$, Coinjection of XneogPan with full-length Xneog1b cRNA increase the mean neurulation stage compared with injection of XneogPan alone. The number of embryos in each condition is given at the top of each graph. Data are represented as mean \pm SEM. ${ }^{* * *} p<0.001 ;{ }^{*} p<0.05$.

the presumptive forebrain and hindbrain (Fig. 1A). During NF elevation (st13-16), Xneog expression intensified in the deep layer cells of the rising NFs (Fig. 1B). Again Xneog was most intense in the anterior neural tube. In addition, the Xneog expression domain had expanded into the midline, with expression appearing in the medial neural plate and presumptive floor plate (Fig. 1 Biii, arrows). In the late neurula, when NF apposition is

occurring (st19), Xneog expression was most intense in the dorsal NFs along the entire neuraxis (Fig. 1C, arrows) while lower expression was observed in the ventral regions. In summary, Xneog is expressed in the deep layer of the neural plate throughout neurulation. It is most prominent in the lateral neural plate which gives rise to the NFs and remains high in the folds as they elevate and migrate toward the midline.

To determine whether Xneog plays a role in Xenopus neurulation we knocked down Xneog expression using antisense MOs. Initially, both cells in two-cell stage Xenopus embryos were injected with $8 \mathrm{ng}$ (16 ng total) of XneogPan MO or ContMO (supplemental Fig. 1, available at www. jneurosci.org as supplemental material) and development was monitored throughout neurulation. Both ContMO- and XneogPan MO-injected embryos developed normally until late gastrulation (Fig. 2A) (st12.5). No defects were observed in blastopore formation or involution in embryos injected with either the ContMO or XneogPan MO. However, from st12.5 onwards, neurulation proceeded at a markedly slower rate in embryos injected with XneogPan MO (morphants) (Fig. 2A). In ContMO embryos, NF apposition (st18) occurred $6 \mathrm{~h}$ after reaching midgastrulation (st11). By the end of this $6 \mathrm{~h}$ period $\sim 90 \%$ of ContMO embryos had reached st 18 . In contrast, within the same time period, only $10-15 \%$ of morphants exhibited a closed neural tube (Fig. 2A-C). We observed that the severity of the delay phenotype varied within each experiment. On average, $6 \mathrm{~h}$ after reaching st $11,11 \%$ of morphants were at st $17,34 \%$ were at st 16 , and $43 \%$ were still at st14-15 (Fig. 2C). While the progression through neurulation in the most severely affected morphants was delayed by $>2 \mathrm{~h}, \mathrm{NF}$ fusion did eventually occur and the embryos survived past st35 (see Fig. 7).

We also observed that anterior neural tube closure appeared to be affected to a greater extent than posterior closure in the morphants (Fig. 2Avii). To further test this hypothesis the amount of injected $\mathrm{MO}$ was increased to $24 \mathrm{ng}$ (12 $\mathrm{ng}$ per cell of two-cell stage embryos) and anterior neural tube closure was scored when $>80 \%$ of ContMO-injected embryos had reached st21-22 (2 h after NF fusion). In the XneogPan MO-injected embryos, an open anterior neural tube was observed in $100 \%$ of morphants compared with $20 \%$ in control embryos $(p<0.001$, Student's $t$ test). However, in all cases, the posterior neural tube had fused. Therefore, the anterior neural tube is more severely affected by knock-down of Xneog when compared with the posterior neural tube. 
Table 1. Mean neurulation stage in XneogPan MO-versus control M0-injected embryos

\begin{tabular}{lll}
\hline Morpholino $^{a}$ & Mean stage $^{b} \pm$ SEM & No. of embryos \\
\hline ContM0 & $17.81 \pm 0.06 p<0.001^{c}$ & 63 \\
XneogPan & $15.89 \pm 0.20$ & 47 \\
XplexATG & $17.91 \pm 0.04 p<0.001$ & 65 \\
XnpnATG & $17.89 \pm 0.04 p<0.001$ & 53 \\
\hline
\end{tabular}

${ }^{a} \mathrm{~A}$ total of $16 \mathrm{ng}$ of each $\mathrm{M} 0$ was injected per embryo.

${ }^{b}$ Mean neurulation stage for embryo cohorts injected with specified M0s. Embryos are staged according to Nieuwkoop and Faber (1994), when $90 \%$ of ContM0-injected embryos exhibit neural fold apposition (st18).

Neurulation stage was analyzed for each condition relative to XneogPan using the Mann-Whitney test.

Table 2. Mean neurulation stage in Xneog1a M0- and 1b M0-injected embryos

\begin{tabular}{llc}
\hline Morpholino $^{a}$ & Mean stage $^{b} \pm$ SEM & No. of embryos \\
\hline ContM0 & $17.94 \pm 0.02$ & 112 \\
XneogPan & $17.09 \pm 0.08 p<0.001^{c}$ & 92 \\
Xneog1aATG & $17.46 \pm 0.09 p<0.001$ & 80 \\
Xneog1bUTR & $16.99 \pm 0.07 p<0.001$ & 109 \\
Xneog1bATG & $17.04 \pm 0.20 p<0.001$ & 25 \\
\hline
\end{tabular}

${ }^{a} \mathrm{~A}$ total of $16 \mathrm{ng}$ of each $\mathrm{M} 0$ was injected per embryo.

${ }^{b}$ Mean neurulation stage for embryo cohorts injected with specified MOs. Embryos are staged according to Nieuwkoop and Faber (1994), when 90\% of ContM0-injected embryos exhibit neural fold apposition (st18).

'Neurulation stage was analyzed for each condition relative to ContM0 using ANOVA and Dunnett's T3 post hoc test.

To demonstrate that defective neural tube closure did not result from a generalized, nonspecific disruption of neurulation resulting from our experimental procedure we injected MOs specific for the semaphorin guidance receptors XplexinA and Xneuropilin1 (Fig. 2B). While $87 \%$ of XneogPan-injected embryos exhibited a delay in neural tube closure, only $10 \%$ of embryos injected with the XplexinA (Xplex) MO or Xneuropilin1 (Xnpn) $\mathrm{MO}$ were delayed. We also calculated the mean neurulation stage for each embryo cohort when $90 \%$ of control embryos had reached st18 (Table 1) and demonstrated that the cohort injected with XneogPan MO (mean stage: st15.9 $\pm 0.2, p<0.001$ ) was significantly delayed when compared with the cohort injected with ContMO (st17.8 \pm 0.1$)$, Xplex MO (st17.9 \pm 0.0$)$, or Xnpn $\mathrm{MO}(\operatorname{st} 17.9 \pm 0.0)$.

There are two distinct Xneog genes (Xneog1a and Xneog1b) in the Xenopus genome (Wilson and Key, unpublished observations) (supplemental Fig. 1, available at www.jneurosci.org as supplemental material). Significant delays in NF apposition were also observed when the XneoglaATG, Xneog1bATG or XneoglbUTR MOs were used (Fig. 2D). In all instances, when control embryos displayed NF apposition (st18), the NFs of Xneog morphants had not yet reached the midline, and were between one to three neurulation stages behind (i.e., st15-17). While the efficiency of each Xneog MO varied, we observed a significant delay $(p<0.001)$ in mean neurulation stage for the cohorts injected with these MOs compared with ContMOinjected cohort (Table 2). The observed differences in the mean neurulation stage resulting from injection of each Xneog MO reflects the severity of the delay. These data indicate that XneoglaATG is less effective in silencing Xneog function than the other MOs tested. In summary, both Xneogla and Xneog1b are required for efficient neurulation in the Xenopus embryo.

The sequence of the 5'UTR target region of the XneogPan MO is derived from Xneog1 $a$ and differs from the corresponding sequence in Xneog $1 b$ by only one nucleotide (supplemental Fig. 1, available at www.jneurosci.org as supplemental material). Therefore, the XneogPan MO is likely to prevent translation from both the Xneog $1 a$ and $1 b$ transcripts. To further demonstrate specificity we performed rescue experiments in which the XneogPan MO was coinjected with a cRNA encoding full-length Xneog1b. We were not able to perform a similar rescue experiment with Xneog $1 a$ as we do not have a full-length Xneog1a cDNA available to us. Nonetheless, coinjection of wild-type Xneog $1 b$ decreased to a small but significant extent the severity of the neurulation delay when compared with that resulting from the injection of the XneogPan $\mathrm{MO}$ alone. Figure $2 E$ shows that coinjection of $X n e o g 1 b$ cRNA with XneogPan MO increased the mean neurulation stage from st16.8 \pm 0.1 (XneogPan MO alone) to st17.2 \pm $0.1(p<0.05)$. That both Xneogla and 1b MOs cause delays in neural tube closure indicates that both Xneogla and $1 \mathrm{~b}$ are required for efficient neurulation. This observation provides an explanation for the small degree of rescue observed with the Xneog $1 b$ cRNA alone.

\section{Xrgma is a ligand for Xneog during neurulation}

In situ hybridization analysis demonstrated strong expression of Xrgma mRNA adjacent to the midline along the entire anteroposterior axis during neurulation (st13-19) where it was localized to the deep cells of the neural plate (Fig. 3). At st13 it was found only in the medial half of the neural plate and was therefore expressed directly adjacent to the domain of Xneog expression in the lateral neural plate (Fig. 3Aiv). During NF elevation (st13-16), the Xrgma expression domain contracted toward the midline (Fig. $3 B)$. At this stage, Xneog expression had expanded toward the midline and therefore overlapped that of Xrgma (Fig. 3Biii, iv). At NF apposition (st19), Xrgma expression became prominent in the ventral neural tube (Fig. $3 C$ ). However, little Xrgma was observed within the strong Xneog expression domain in the dorsal neural tube (Fig. 3Ciii, iv). Therefore, Xrgma is expressed adjacent to the midline and partially overlaps the Xneog domain during neurulation.

An antisense MO knock-down strategy was then used to determine whether Xrgma was required for neural tube closure. Figure $4 A$ demonstrates that injection of the XrgmaATG or XrgmaUTR MOs (16 ng total) (supplemental Fig. $1 B$, available at www.jneurosci.org as supplemental material) at the two-cell stage resulted in a significant delay in neural tube closure $(p<$ $0.001, p<0.05$, respectively). The severity of delay induced by the XrgmaATG MO was equivalent to that seen with the XneogPan MO (Fig. 2A), while injection of the XrgmaUTR MO produced a less severe phenotype. However, we observed a significant delay in mean neurulation stage for the embryo cohort injected with either Xrgma MO compared with the ContMO-injected cohort (Table 3). To further demonstrate that Xrgma functions as a ligand for Xneog during neurulation, we coinjected a hypomorphic dose of XneogPan MO with XrgmaATG MO or ContMO (total of 8 ng of each MO) (Fig. $4 B$ ). While coinjection of XneogPan MO (+ ContMO) resulted in delayed NF fusion in $30 \%$ of embryos, no significant delay was observed upon coinjection of XrgmaATG MO (+ ContMO). However, coinjection of XneogPan $\mathrm{MO}$ and XrgmaATG MO resulted in a significant increase in the number of delayed embryos (71\%) above that seen when either MO was injected with the control MO $(p<0.001)$. No additional phenotypic abnormalities were observed upon coinjection of the receptor and ligand MOs. The increase in phenotypic severity could not be attributed to nonspecific interactions as coinjection of XneogPan MO and the Xnpn MO did not enhance the neurulation delay (data not shown).

To further confirm that knock-down of Xneog and Xrgma perturbs neurulation in an equivalent manner we plotted the time taken (post st12.5) for embryos to reach key neurulation stages (Fig. 4C). All embryos injected with either the XrgmaATG 

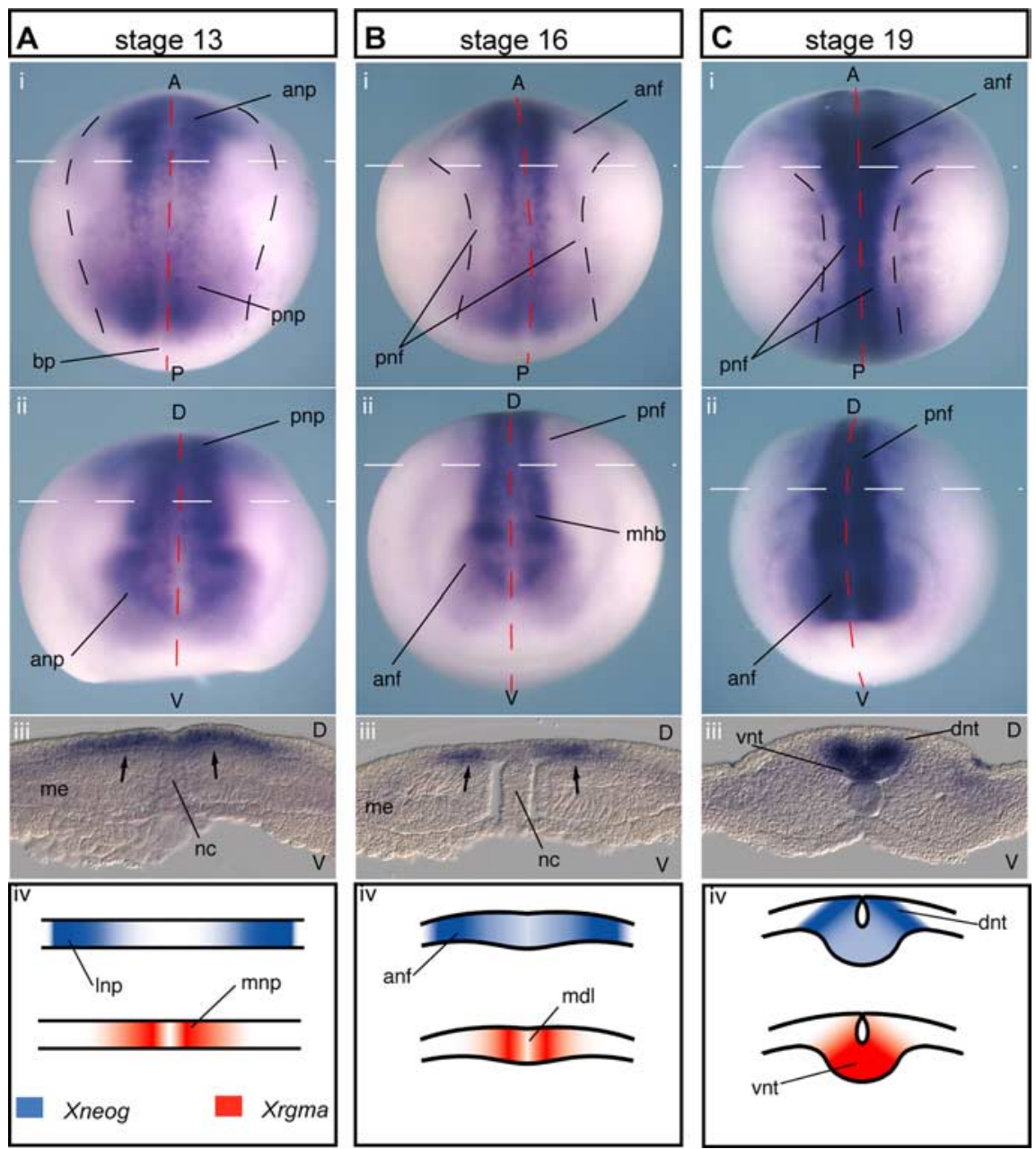

Figure 3. Xrgma expression in the deep layer of the medial neural plate. Xrgma is expressed $(\boldsymbol{A})$ in the deep layer of the st13 medial posterior neural plate (Ai, Aiii, arrows) and the medial anterior neural plate (Aii), (B) between the midline and the posterior NFs ( $\boldsymbol{B i}$, Biii, arrows), as well as between the midline and the anterior NFs ( $\boldsymbol{B i i})$ of the st16 neurula, and $(\boldsymbol{C})$ in the st19 ventral neural tube (Ci, Cii, Ciii). (i), Dorsal view; (ii), frontal view; (iii), transverse view at the level of the dashed white line in above panels; (iv), schematic of complementary Xneog and Xrgma expression domains. Inp, Lateral neural plate; mdl, midline; mnp, medial neural plate.

or XneogPan MO displayed a wild-type phenotype before st12.5, and all Xneog and Xrgma morphants reached st12.5 at the same time as control embryos. However from st12.5 onwards, the progression through neurulation was markedly slower for morphants compared with control embryos. Moreover, the rate of neurulation in both Xneog and Xrgma morphants was equivalent. Therefore, we conclude that Xrgma interacts with Xneog during neurulation to promote neural tube closure.

\section{Xneog and Xrgma are required for NF elevation}

To identify the morphological defects underlying failure of neural tube closure, we injected $12 \mathrm{ng}$ of XneogPan MO or ContMO unilaterally into one cell of two-cell stage embryos and immunolabeled at st 17 with an antibody specific for $\beta$-tubulin. The temporal progression of NF elevation, neural plate bending and NF migration in embryos unilaterally injected with the ContMO was equivalent on the ContMO (Fig. $5 A$, right, arrow) and wild-type side (Fig. 5A, left). In addition, no disruption of neural plate morphology was observed on the ContMO-injected side. In contrast, the unilateral injection of XneogPan $\mathrm{MO}$ resulted in a obvious perturbation of NF elevation at both the anterior and posterior levels (Fig. 5 B,I, right, arrows) when compared with the uninjected, wild-type side (Fig. 5B, I, left). Figure $5 B$ clearly demonstrates that while the anterior NF on the wild-type side had undergone appropriate elevation, the height of the fold on the morphant side was markedly reduced, leading to a broadening of the fold in the lateral direction (Fig. 5B, arrow). Similarly, failure of NF elevation was observed on the morphant side of the posterior neural plate (Fig. 5I, arrow). Therefore, loss of Xneog results in inefficient NF elevation along the entire neuraxis. Unilateral injections of the XrgmaATG MO produced a similar, albeit less severe, NF elevation phenotype (data not shown), confirming that Xrgma is the Neogenin ligand promoting NF elevation. It is important to note that, despite the suppression of NF elevation in the absence of Xneog or Xrgma, the hinge-points formed correctly (Fig. 5B, arrowheads) and the distance between the midline and hinge-point was equivalent to that on the wild-type side, suggesting that both apical constriction and convergent extension were still operative.

\section{Loss of Xneog perturbs deep layer cell polarity by disrupting the microtubule cytoskeleton}

From the start of neurulation (st13) until after neural tube closure (st22) cells of the deep layer are elongated in an apicobasal direction (initially the apicobasal plane equates to the dorsoventral plane of the neural plate) and are tightly apposed in wild-type embryos. Close examination of the unilaterally injected st 17 embryos immunolabeled with the anti- $\beta$-tubulin antibody revealed that while the deep cells in the wild-type anterior NF were elongated in the apicobasal direction (Fig. $5 C$ ), those on the XneogPaninjected side had lost their apicobasal polarity and displayed a rounded morphology (Fig. 5D, arrowheads). Higher magnification revealed that the microtubule (MT) cytoskeleton in these cells was disrupted. In the presence of the XneogPan MO, $\beta$-tubulin immunoreactivity was found in punctate aggregates or dispersed throughout the cytoplasm of the deep cells, indicating depolymerization and fragmentation of the MT filaments (Fig. $5 D, F)$. In contrast, MT filaments in wild-type deep cells formed a tight mesh of fibers adjacent to the plasma membrane (Fig. $5 C, E)$. Disruption of the MT cytoskeleton was also observed when the XrgmaATG MO was unilaterally injected (data not shown). This phenotype is highly consistent, as it was also observed in embryos when lower doses of XneogPan or XrgmaATG MOs were bilaterally injected ( $8 \mathrm{ng}$ into each cell of two-cell stage embryos). Together, these observations suggest that XneogXrgma interactions establish apicobasal polarity in the deep layer cells by regulating the MT network.

Within polarized neuroepithelium, tight junctions are found adjacent to the apical surface of the cell. Therefore, localization of junctional proteins (e.g., ZO-1) to the tight junction indicates that correct apicobasal polarity has been established (Nelson, 


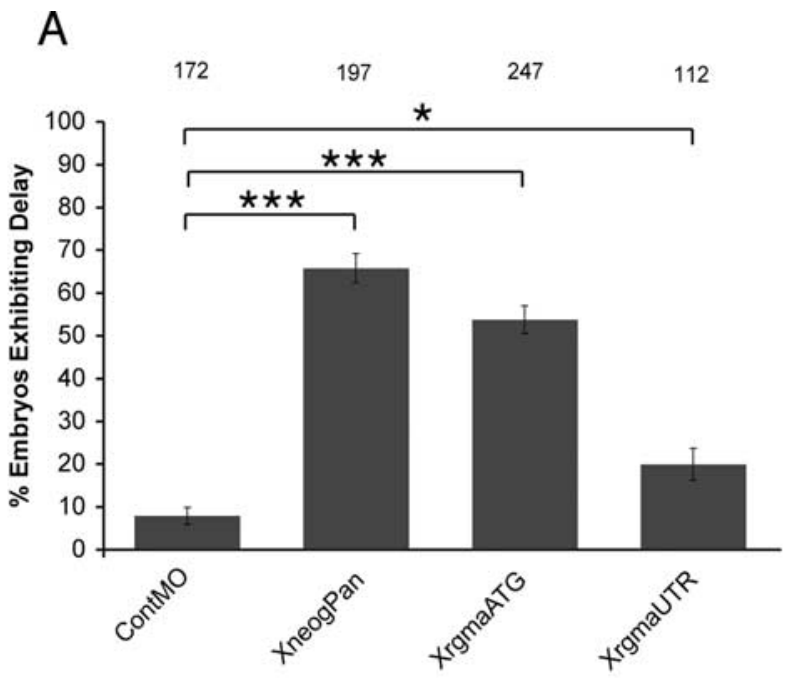

B

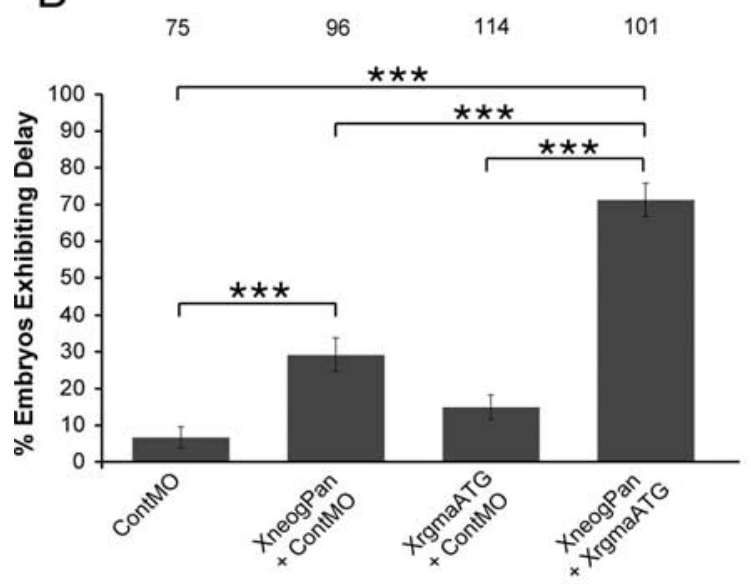

C

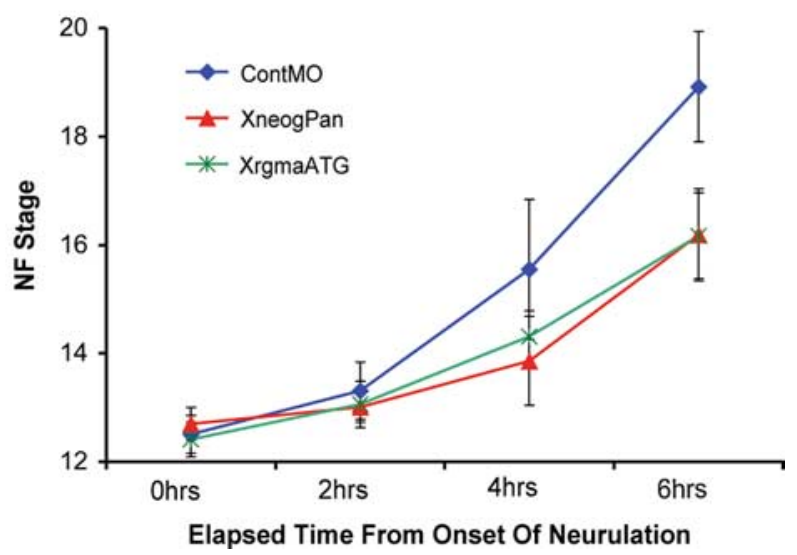

Figure 4. Loss of Xrgma results in neural tube closure defects. A, Injection of $16 \mathrm{ng}$ of $\mathrm{Xrg}$ maATG MO or XrgmaUTR M0 produces a neurulation delay phenotype. $\boldsymbol{B}$, Coinjection of $8 \mathrm{ng}$ of XneogPan and XrgmaATG MOs enhances the delay in neurulation. Data are represented as mean \pm SEM. C, XneogPan MO and XrgmaATG MO generate equivalent decreases in the rate of progression through neurulation. Data are represented as mean \pm SD.

2003). We wished to confirm the requirement for Neogenin in the establishment of apicobasal polarity during neurulation by investigating the distribution of ZO-1 in our Xenopus morphants. However, we were not able to identify an antibody that recog-
Table 3. Mean neurulation stage in Xrgma M0-injected embryos

\begin{tabular}{lll}
\hline Morpholino $^{a}$ & Mean stage $^{b} \pm$ SEM & No. of embryos \\
\hline ContM0 & $17.92 \pm 0.02$ & 172 \\
XneogPan & $16.81 \pm 0.08 p<0.001^{c}$ & 197 \\
XrgmaATG & $17.11 \pm 0.06 p<0.001$ & 247 \\
XrgmaUTR & $17.76 \pm 0.05 p=0.002$ & 112 \\
\hline
\end{tabular}

${ }^{a} \mathrm{~A}$ total of $16 \mathrm{ng}$ of each M0 was injected per embryo.

${ }^{b}$ Mean neurulation stage for embryo cohorts injected with specified MOs. Embryos are staged according to Nieuwkoop and Faber (1994), when 90\% of ContM0-injected embryos exhibit neural fold apposition (st18).

CNeurulation stage was analyzed for each condition relative to ContM0 using the Mann-Whitney test.

nized the ZO-1 orthologue in Xenopus laevis borealis embryos. Instead, we examined ZO-1 distribution in zebrafish embryos, since the disrupted morphology of the zebrafish neural tube in the absence of Neogenin (Mawdsley et al., 2004) exactly phenocopies that observed in the Xenopus morphants. Like most vertebrates, the zebrafish neural plate comprises a single layer of columnar epithelium (Papan and Campos-Ortega, 1994; Lowery and Sive, 2004). During neurulation, the zebrafish neural plate rolls up and condenses into a solid mass of cells (the neural keel) which then sinks into the underlying mesoderm to form a solid rod of neuroepithelium (the neural rod) (Geldmacher-Voss et al., 2003). Lumination then progresses in a ventral to dorsal direction (Schmitz et al., 1993) in a similar manner to Xenopus trunk relumination (Davidson and Keller, 1999). Despite the morphological differences between Xenopus and zebrafish neurulation, neural keel and neural rod formation are also reliant on the establishment of apicobasal polarity and radial intercalation (for review, see Lowery and Sive, 2004).

To examine the effect of loss of Neogenin on ZO-1 distribution in the neuroepithelium of zebrafish morphants we injected a control MO (zfcontMO) or a MO directed to the ATG sequence of zebrafish Neogenin (zfneogATG) into the yolk sac of two-cell stage embryos. We then visualized the morphology of the neuroepithelium within the neural rod by staining the plasma membranes with the lipophilic dye, DiI (Fig. 6). In zfcontMO-injected embryos ZO-1 protein was localized to the tight junctions lying immediately below the apical surface of the neuroepithelial cells at the midline (Fig. 6A). In striking contrast, the morphology of the neural rod was severely disrupted in zfneogATG MO-injected embryos. No obvious midline could be visualized in the morphants and neuroepithelial cells were rounded and no longer aligned in an apicobasal orientation (Fig. 6 B, C). Moreover, ZO-1 immunoreactivity was no longer restricted to one region of the plasma membrane, but was found distributed around the entire cell surface (Fig. 6B,C). Therefore, in the absence of Neogenin the distribution of ZO-1 was not apically polarized, demonstrating that the neuroepithelial cells had lost their apicobasal polarity.

\section{Loss of Xneog leads to failure of radial intercalation and inefficient superficial cell wedging}

In the Xenopus morphants (Fig. 5) we further observed that the inability of deep layer cells to maintain an apicobasally orientated elongated shape resulted in the failure of radial intercalation with the overlying superficial cell layer in the anterior neural tube. Figure 5 shows that, around the hinge-point of the wild-type side, the apical membranes of the deep cells (Fig. $5 G$, arrowheads) had intercalated with the superficial layer forming close contacts with adjacent wedge-shaped superficial cells (Fig. 5G, asterisks). In striking contrast, the rounded deep cells (Fig. $5 \mathrm{H}$, arrowheads) on the morphant side had not interdigitated with the superficial cells (Fig. $5 H$, asterisks), leaving both layers only loosely associ- 

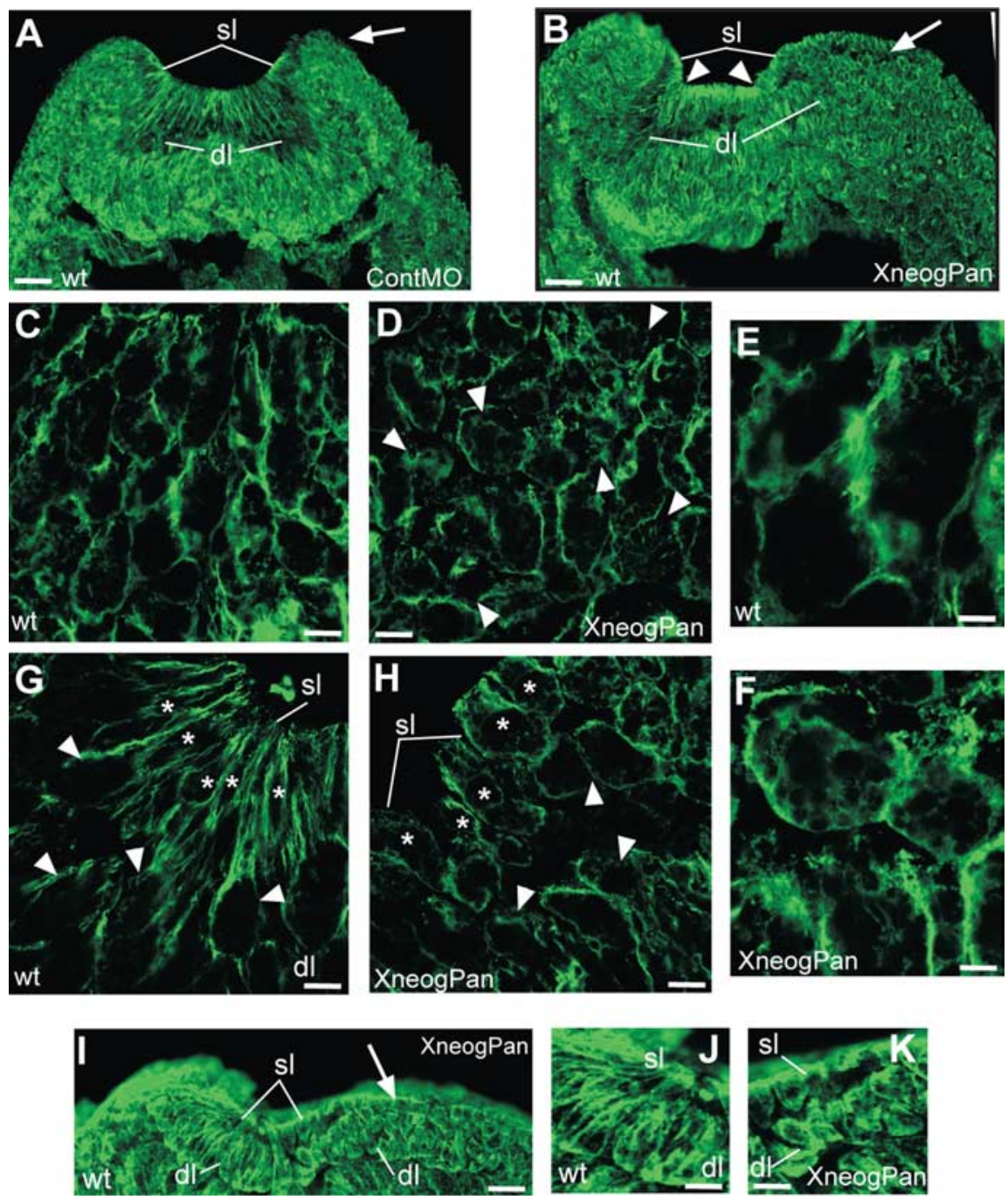

Figure 5. Loss of Xneog perturbs NF elevation and cell polarity. Transverse sections of st17 embryos immunostained with the anti- $\beta$-tubulin antibody, E7. $A$, Injection of ContM0 into one cell of two-cell stage embryos did not affect anterior NF elevation on the ContM0 (right side, arrow) or the wild-type (wt) side (left side) of the embryo. $\boldsymbol{B}$, Injection of XneogPan M0 disrupts anterior NF elevation on the morphant side of the embryo (right side, arrow). Hinge-points are indicated by arrowheads. $\boldsymbol{C}-\boldsymbol{F}$, Higher magnification images of the deep layer ( $\mathrm{dl})$ in $\boldsymbol{B}$ on the wild-type side $(\boldsymbol{C}, \boldsymbol{E})$ and XneogPan M0 side $(\boldsymbol{D}, \boldsymbol{F})$ where deep cells have lost their apicobasal polarity (arrowheads). $\mathbf{G}-\boldsymbol{H}$, Higher magnification images of the deep (arrowheads) and superficial layers (sl, asterisks) in $\boldsymbol{B}$ at the hinge-point on the wild-type side ( $\boldsymbol{G}$ ) and the XneogPan M0 side $(\boldsymbol{H})$. $\boldsymbol{I}-\boldsymbol{K}$, Posterior NF elevation is inhibited on the XneogPan M0 side (I, arrow; $\boldsymbol{K}$ ) but not the wild-type side (I, left side; $\boldsymbol{J})$. Scale bars: $\boldsymbol{A}, \boldsymbol{B}, \boldsymbol{I}, 39 \mu \mathrm{m} ; \boldsymbol{C}, \boldsymbol{D}, \boldsymbol{G}, \boldsymbol{H}, 2 \mu \mathrm{m} ; \boldsymbol{E}, \boldsymbol{F}$, $1 \mu \mathrm{m} ; \boldsymbol{J}, \boldsymbol{K}, 16 \mu \mathrm{m}$.

ated. Moreover, while some degree of apical constriction had occurred in these superficial cells, they were significantly less wedge-shaped and less elongated than those on the wild-type side. Also, while $\beta$-tubulin had accumulated under the apical surface in the morphant superficial cells, long apicobasally directed MT filaments originating from the apical region (Karfunkel, 1971; Burnside, 1973; Lee et al., 2007) were only seen in wild-type cells (Fig. 5, compare $G, H$ ). Knock-down of Xneog in the posterior neurula also resulted in loss of the apicobasally orientated elongated shape of the deep layer cells, failure of radial intercalation, and loss of apical constriction in superficial cells (Fig. 5, compare $J, K$ ). Therefore, our data indicate that efficient apical constriction and elongation of superficial cells is reliant on appropriate interaction with the intercalating deep cells. Since Xneog and Xrgma are not expressed in the superficial layer, the disruption to this layer is a non-cell-autonomous effect, secondary to the disruption of deep layer cell polarity and intercalation.
These observations indicate that apicobasal polarization of the deep layer cells is essential for radial intercalation. Moreover, our data suggest that superficial cell wedging is potentiated by intimate contact with appropriately polarized deep cells.

\section{Xneog and Xrgma are required for apicobasal polarity of the pseudostratified epithelium in the neural tube}

While knock-down of Xneog or Xrgma using a total of $16 \mathrm{ng}$ of MO per embryo resulted in a significant delay in NF elevation, the NFs did eventually meet and fuse at the midline to form a neural tube. To determine whether loss of Xneog and Xrgma continued to perturb cell polarity within the pseudostratified epithelium of the neural tube after closure (st35) we used the Xen 1 antibody which recognizes an unknown epitope on the cell surface of Xenopus neuroepithelial cells (Ruiz i Altaba, 1994). While the pseudostratified epithelium of the ContMO neural tube developed normally (Fig. 7A), the Xneog and Xrgma morphant neural tubes displayed a severely perturbed neuroepithelium with a smaller, misshapen lumen (Fig. $7 B, C$, respectively). Within the morphant neural tube, the neuroepithelial cells had detached from the apical and basal surfaces, become rounded, and were distributed in a disorganized manner throughout the structure (Fig. 7Bii,iii, Cii,iii). Counterstaining with DAPI revealed many pyknotic nuclei within the neuroepithelia and luminal spaces of the Xneog and Xrgma morphants, indicating that large numbers of cells were undergoing cell death (Fig. $7 \mathrm{Bi}, \mathrm{Ci}$ ). No pyknotic cells were observed in the control neural tube. These neural tube defects were never observed in embryos injected with the XplexinA or Xneuropilin1 MOs (data not shown).

To confirm specificity, we performed a rescue experiment in which the XneogPan MO was coinjected with a cRNA encoding full-length Xneoglb. When carrying out similar experiments to rescue the neurulation delay phenotype (Fig. 2E), we found that coinjection of Xneog $1 b$ cRNA only rescued this phenotype to a small extent. To examine the ability of Xneog1b to rescue the aberrant morphology of the st35 neural tube we examined the neural tube of embryos that exhibited a neurulation delay in the presence of the coinjected XneogPan MO and the Xneog $1 b$ cRNA (Fig. $8 C$ ). That is, embryos coinjected with XneogPan $\mathrm{MO}+$ Xneog1b cRNA that were found to be a st13-st16 when $90 \%$ of ContMO injected embryos had reached st18 were examined. Figure $8 C$ shows that Xneog $1 b$ cRNA was able to almost fully rescue the morphology of the st 35 neuroepithelium after the neural tube had eventually closed. This suggests that the maintenance of neuroepithelial morphology in the st35 neural tube is predominantly dependent on Xneoglb.

In summary, we have demonstrated that Xrgma-Xneog inter- 


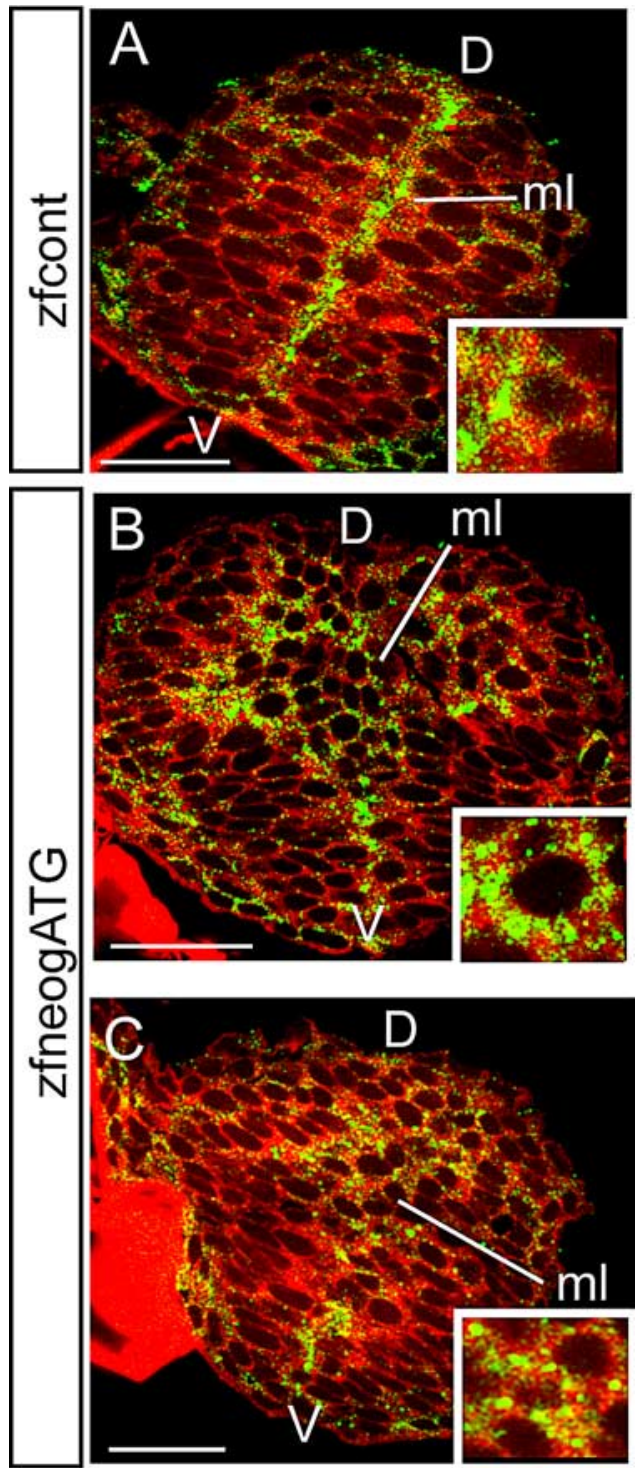

Figure 6. Loss of Neogenin disrupts localization of Z0-1 to the apical domain of neuroepithelial cells in the zebrafish neural rod. $\boldsymbol{A}, \mathbf{2 0}$-1 protein (green) is restricted to a domain adjacent to the apical surface of neuroepithelial cells at the midline $(\mathrm{ml})$ of the neural rod of embryos injected with zfcont MO. $\boldsymbol{B}, \boldsymbol{C}$, Injection of the zfneogATG M0 resulted in loss of an obvious midline. Neuroepithelial cells were rounded and no longer aligned in an apicobasal orientation. In addition, 20-1 immunoreactivity was distributed around the entire cell surface. Insets are high-magnification images of cells exhibiting a polarized distribution of 20-1 in control embryos $(\boldsymbol{A})$ or a nonpolarized distribution of Z0-1 in morphant cells $(\boldsymbol{B}, \boldsymbol{C})$. The lipophilic dye, Dil (red) was used to visualize the cell shape of the neuroepithelium. Scale bars: $A-C, 20 \mu \mathrm{m}$.

actions maintain deep cell morphology within the neural plate during NF elevation and are therefore required for successful neural tube closure along the entire neuraxis, with the anterior neural tube being most sensitive to loss of this receptor-ligand pair. We also show that RGMa and Neogenin are essential for the establishment of apicobasal polarity of the pseudostratified neuroepithelium within the neural tube. Moreover, our data suggests that correct neurulation requires both Xneogla and Xneog1b, while Xneog $1 \mathrm{~b}$ is the predominant form in the neuroepithelium after neural tube closure.

\section{Discussion}

Over the past decade, the molecular pathways regulating convergent extension (the noncanonical Wnt/PCP pathway) and neural plate bending (Shroom3, Sonic hedgehog, BMP-2 pathways) have been identified (Ybot-Gonzalez et al., 2002, 2007; Wallingford, 2005; Kibar et al., 2007). However, as yet, no signaling pathway has been linked to NF elevation. Here we show that XneogXrgma interactions are essential for effective NF elevation. We provide evidence that Xneog is required to maintain the integrity of the MT network within the deep layer cells of the neural plate. We also demonstrated that Xneog-dependent regulation of MTs within the deep cells is essential for their radial intercalation with the overlying superficial cells which is a prerequisite for NF elevation. A requirement for Xneog and Xrgma in the establishment of apicobasal polarity and therefore epithelialization of the pseudostratified neuroepithelium within the neural tube was also demonstrated.

\section{Neogenin controls the MT cytoskeleton}

At the onset of Xenopus neurulation (st13) the deep layer cells elongate such that they are orientated in the dorsoventral plane of the neural plate (this will become the apicobasal plane during neural groove formation) (Schroeder, 1970; Karfunkel, 1971; Keller et al., 1992; Davidson and Keller, 1999). At st13 the MTs are randomly distributed throughout the deep cell cytoplasm (Karfunkel, 1971). However, as NF elevation proceeds, the MT filaments align along the apicobasal axis of the deep cells as they undergo radial intercalation. Therefore, active reorientation of the MT network in the direction of migration accompanies the initiation of deep cell intercalation. Examination of the MT network of deep cells in the Xneog or Xrgma morphants using an antibody to $\beta$-tubulin revealed that, in the absence of Neogenin activity, tubulin had dispersed throughout the cytoplasm, indicating depolymerization of the MTs. These observations suggest that Xneog-Xrgma interactions establish the apicobasally orientated shape of the deep layer cells by regulating the MT network. The hypothesis that Neogenin is important in the establishment of apicobasal polarity is supported by the perturbation in apical localization of the polarity marker, ZO-1, in the neuroepithelium of zebrafish injected with the zfneogATG MO (Fig. 6B, C).

That the integrity of an apicobasally orientated MT network is essential for radial intercalation and NF elevation is supported by an early study on the role of MTs in Xenopus neurulation (Karfunkel, 1971). Strikingly, the phenotype observed after exposure of embryos throughout neurulation to the tubulin polymerization inhibitor, vinblastine, exactly phenocopies the NF and neural tube disruptions observed in our Xneog and Xrgma morphants. Therefore, disruption of the MT network by drug treatment or by silencing of Neogenin activity results in equivalent perturbations in deep cell morphology, loss of NF elevation and neural tube morphology, supporting the hypothesis that Neogenin orientates the MT network of intercalating deep layers, thereby establishing correct apicobasal polarity. At present, we do not know how Neogenin regulates the MT network. However, it is known that the neural-specific MT associated protein, MAP1B, lies downstream of the Netrin receptor DCC, and is phosphorylated by Netrin-dependent activation of GSK3 and CDK5 (Del Río et al., 2004). Thus, Neogenin may also control MT activity via a similar pathway.

\section{Neogenin-dependent radial intercalation drives NF elevation} Our study has revealed the dependence of NF elevation on Neogenin-driven radial intercalation. We observed that the aberrant morphology of the deep layer cells prohibited intercalation with the overlying superficial layer. In the Xenopus neurula, it is the lateral and intermediate regions of the neural plate that un- 


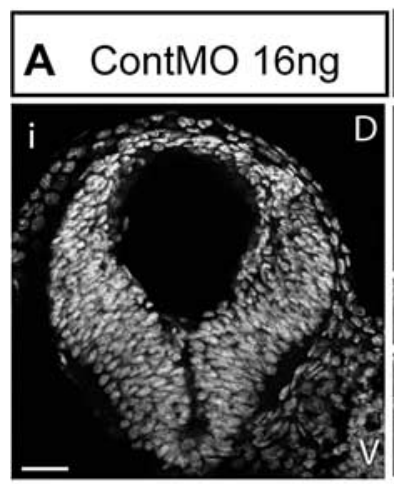

\section{B XneogPan 16ng}
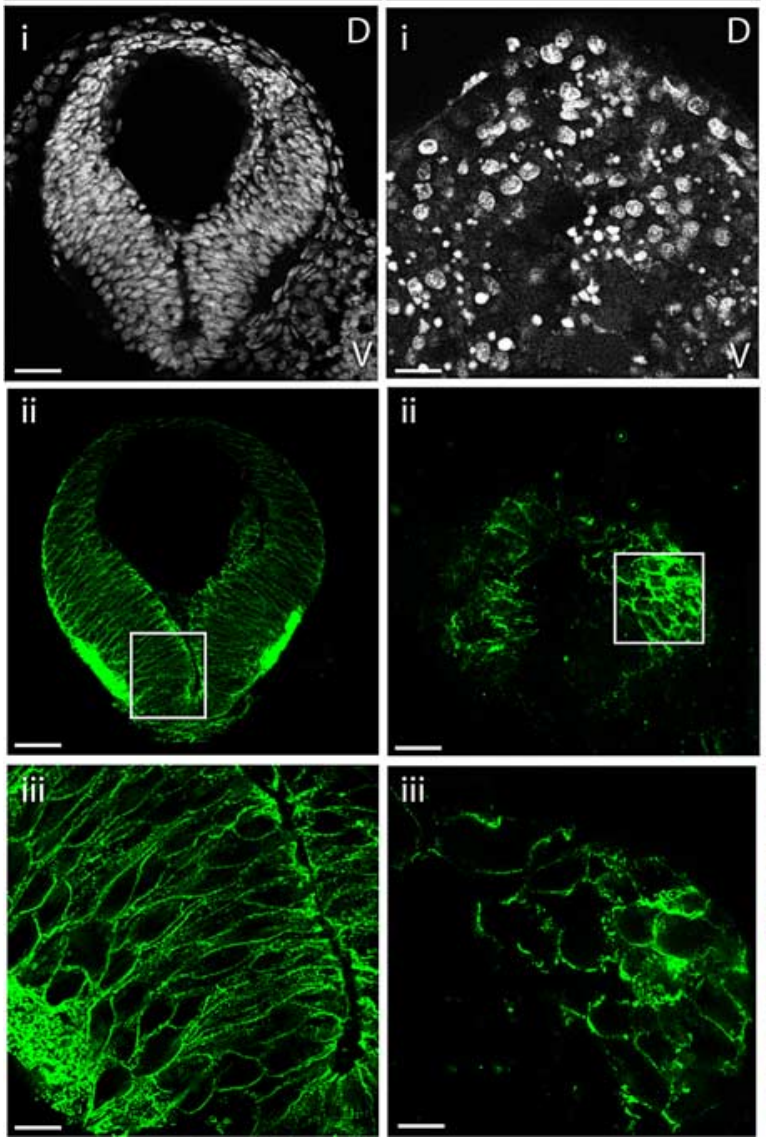

establishment of apicobasal polarity in epithelial cells (Haigo et al., 2003; Lee et al., 2007). However, the mechanism by which apicobasal polarity is initially established in superficial cells has not been elucidated. Our data indicate that constriction of superficial cells is potentiated by contact with the intercalating deep cells. Therefore, we propose that Neogenin activity, via its promotion of deep cell radial intercalation, also regulates superficial cell polarity and, consequently, apical constriction.
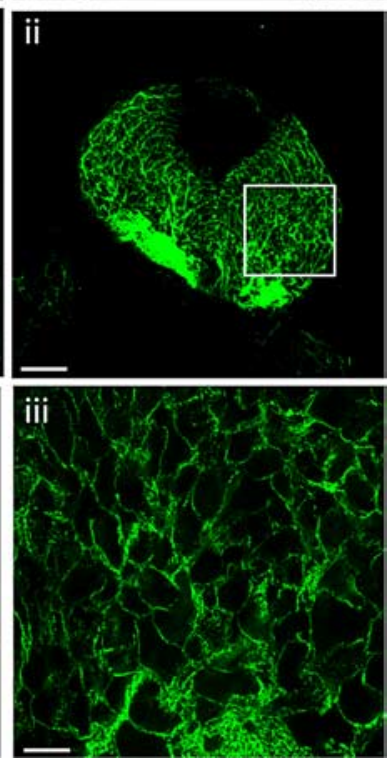

Figure 7. Xneog and Xrgma are required for the establishment of the neuroepithelium of the neural tube. Transverse sections of the st35 anterior neural tube labeled with DAPI (Ai, Bi, Ci) and the Xen1 antibody (Aii, iii, Bii, iii, Cii, iii). Injection of XneogPan MO $(\boldsymbol{B})$ or XrgmaATG MO $(\boldsymbol{C})$ but not ContMO $(\boldsymbol{A})$ disrupts the neuroepithelium of the neural tube. Aiii, $\boldsymbol{B}$ iiii and $\mathbf{C i i i}$ are higher magnification of images of boxed areas in Aii, Bii and Cii, respectively. Scale bars: Ai-Cii, $29 \mu \mathrm{m} ;$ Aiii-Ciii, $8 \mu \mathrm{m}$.

dergo elevation to form the NFs (Schroeder, 1970, 1971; Davidson and Keller, 1999). Intercalation of deep and superficial cells proceeds from lateral to medial, with interdigitation in the lateral and intermediate regions of the neural plate increasing threefold as the NFs elevate and migrate toward the midline (Hartenstein, 1989). We have shown that intense Xneog mRNA expression is initially localized to the lateral deep layer of the neural plate and then expands into the intermediate deep layer as NF elevation proceeds. Therefore, Neogenin is present in those cells actively participating in radial intercalation and NF elevation. Together these observations indicate that Neogenin activity in the deep cell layer promotes radial intercalation by regulating apicobasal polarity through the MT network.

Unexpectedly, loss of Neogenin also disrupted the integrity of the superficial layer. These cells had undergone some degree of apical constriction as a high concentration of tubulin was localized under the apical membrane (Karfunkel, 1971; Burnside, 1973; Lee et al., 2007) and obvious hinge-points were generated in the mutants, indicating that apical constriction had occurred, albeit less efficiently. Since Xneog and Xrgma are not expressed in the superficial layer, this disruption is non-cell-autonomous and secondary to the disruption of the deep cell layer. Recently, the cytoskeletal organizing protein, Shroom3, has been shown to be necessary and sufficient to trigger apical constriction in superficial cells. Shroom 3 activity is strictly dependent upon the prior faces or each other. At NF apposition, Xneog mRNA is present in a ventral-low, dorsal-high gradient throughout the early neural tube and is therefore expressed by deep cells actively undergoing radial interaction and epithelialization. From the early phase of NF elevation through to NF fusion, loss of Neogenin in deep cells of the lateral and intermediate neural plate disrupts MT integrity and subsequently intercalation with the superficial layer. Thus the st 35 neural tube phenotype seen in our morphants provides strong evidence that sustained RGMa-Neogenin activity is also essential for deep cell radial interaction and epithelialization during, and subsequent to, NF fusion. Further support for this hypothesis comes from our zebrafish study where knock-down of Neogenin produced severe neural tube defects and loss of neuroepithelial morphology identical to that seen in our Xenopus morphants (Fig. 6) (Mawdsley et al., 2004). As for Xenopus, zebrafish neurulation is reliant on the establishment of apicobasal polarity and radial intercalation (Lowery and Sive, 2004; Hong and Brewster, 2006).

\section{RGMa is the Neogenin ligand during neurulation}

RGMa is linked to the plasma membrane by a glycosylphosphatidylinositol moiety and can be cleaved to produce a soluble isoform (Matsunaga et al., 2004; Niederkofler et al., 2004). Therefore, it can act as either a short- or long-range morphogenic factor. Throughout neurulation the highest level of Xneog expres- 
sion is found in the lateral deep cell layer underlying the rising NFs, while the most intense Xrgma expression domain is restricted to the more medial deep cells (Fig. 3). This complementary expression pattern suggests that at the onset of neurulation, the relevant form of RGMa may be the cleaved isoform which is capable of establishing a morphogenic gradient. As the NFs elevate, the Xneog expression domains expand medially resulting in overlap between the Xneog and Xrgma domains. This raises the possibility that as the Neogeninpositive deep cells intercalate, the membrane-bound RGMa isoform on the adjacent cell may act to potentiate cell-cell contact. To date, RGMa has been shown to act as a chemorepulsive axon guidance cue (Rajagopalan et al., 2004; Wilson and Key, 2006). In contrast, our data suggest that the outcome of RGMa-Neogenin interactions in the context of neurulation is the establishment or maintenance of cell polarity and epithelial integrity. A similar situation occurs during mouse mammary gland development, where Netrin-1Neogenin interactions are required to stabilize the structure of the developing mammary epithelium (Srinivasan et al., 2003; De Vries and Cooper, 2008). That RGMa is important for neural tube closure is further supported by the occurrence of exencephaly in RGMa loss-of-function mouse embryos (Niederkofler et al., 2004).

In summary, we show that RGMa and Neogenin are essential for efficient NF elevation and establishment of the pseudostratified epithelium of the neural tube via their ability to regulate apicobasal polarity and radial intercalation.

\section{References}

Burnside B (1973) Microtubules and microfilaments in amphibian neurulation. Am Zool 13:989-1006.

Connor RM, Key B (2002) Expression and role of Roundabout-1 in embryonic Xenopus forebrain. Dev Dyn 225:22-34.

Copp AJ (2005) Neurulation in the cranial region-normal and abnormal. J Anat 207:623-635.

Davidson LA, Keller RE (1999) Neural tube closure in Xenopus laevis involves medial migration, directed protrusive activity, cell intercalation and convergent extension. Development 126:4547-4556.

Del Río JA, González-Billault C, Ureña JM, Jiménez EM, Barallobre MJ, Pascual M, Pujadas L, Simó S, La Torre A, Wandosell F, Avila J, Soriano E (2004) MAP1B is required for Netrin 1 signaling in neuronal migration and axonal guidance. Curr Biol 14:840-850.

De Vries M, Cooper HM (2008) Emerging roles for neogenin and its ligands in CNS development. J Neurochem 106:1483-1492.

Geldmacher-Voss B, Reugels AM, Pauls S, Campos-Ortega JA (2003) A 90degree rotation of the mitotic spindle changes the orientation of mitoses of zebrafish neuroepithelial cells. Development 130:3767-3780.

Haigo SL, Hildebrand JD, Harland RM, Wallingford JB (2003) Shroom induces apical constriction and is required for hingepoint formation during neural tube closure. Curr Biol 13:2125-2137.

Hartenstein V (1989) Early neurogenesis in Xenopus: the spatio-temporal pattern of proliferation and cell lineages in the embryonic spinal cord. Neuron 3:399-411.

\section{B XneogPan 12ng C $\begin{gathered}\text { XneogPan 12ng } \\ \text { Xneog1b 4ng }\end{gathered}$}
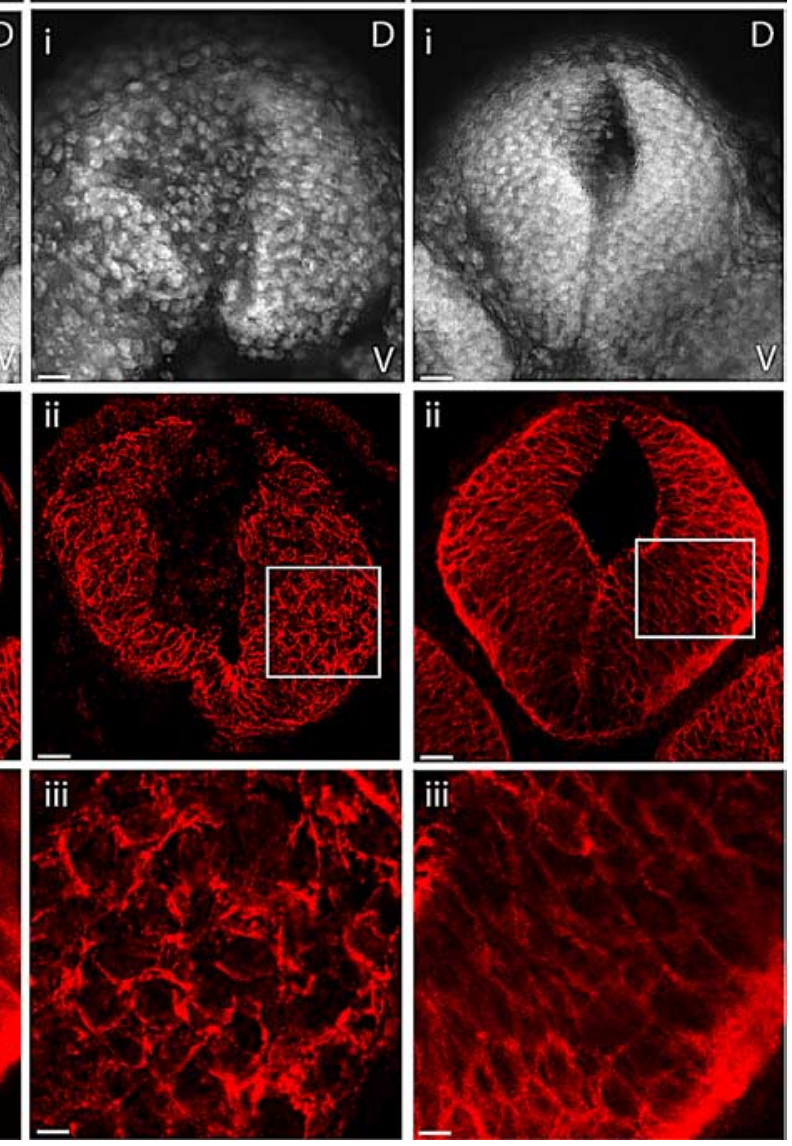

Coinjection of Xneog1b cRNA with XneogPan MO rescues the formation of the neuroepithelium in the neural tube. with XneogPan MO rescues neural tube morphology. Aiii, Biii and Ciiiare higher magnification of images of boxed areas in $\mathbf{A i i , B i}$ and Cii, respectively. Scale bars: Ai-Cii, $39 \mu \mathrm{m}$; Aiii-Ciii, $17 \mu \mathrm{m}$.

Hildebrand JD, Soriano P (1999) Shroom, a PDZ domain-containing actinbinding protein, is required for neural tube morphogenesis in mice. Cell 99:485-497.

Hong E, Brewster R (2006) N-cadherin is required for the polarized cell behaviors that drive neurulation in the zebrafish. Development 133:3895-3905.

Kang JS, Yi MJ, Zhang W, Feinleib JL, Cole F, Krauss RS (2004) Netrins and neogenin promote myotube formation. J Cell Biol 167:493-504.

Karfunkel P (1971) The role of microtubules and microfilaments in neurulation in Xenopus. Dev Biol 25:30-56.

Keeling SL, Gad JM, Cooper HM (1997) Mouse Neogenin, a DCC-like molecule, has four splice variants and is expressed widely in the adult mouse and during embryogenesis. Oncogene 15:691-700.

Keller R (2002) Shaping the vertebrate body plan by polarized embryonic cell movements. Science 298:1950-1954.

Keller R, Shih J, Sater A (1992) The cellular basis of the convergence and extension of the Xenopus neural plate. Dev Dyn 193:199-217.

Kibar Z, Capra V, Gros P (2007) Toward understanding the genetic basis of neural tube defects. Clin Genet 71:295-310.

Lecuit T, Lenne PF (2007) Cell surface mechanics and the control of cell shape, tissue patterns and morphogenesis. Nat Rev Mol Cell Biol 8:633-644.

Lee C, Scherr HM, Wallingford JB (2007) Shroom family proteins regulate gamma-tubulin distribution and microtubule architecture during epithelial cell shape change. Development 134:1431-1441.

Lowery LA, Sive H (2004) Strategies of vertebrate neurulation and a reevaluation of teleost neural tube formation. Mech Dev 121:1189-1197. 
Matsunaga E, Tauszig-Delamasure S, Monnier PP, Mueller BK, Strittmatter SM, Mehlen P, Chédotal A (2004) RGM and its receptor neogenin regulates neuronal survival. Nat Cell Biol 6:749-755.

Mawdsley DJ, Cooper HM, Hogan BM, Cody SH, Lieschke GJ, Heath JK (2004) The netrin receptor, neogenin, is required for neural tube formation and somitogenesis in zebrafish. Dev Biol 269:302-315.

Nelson WJ (2003) Adaptation of core mechanisms to generate cell polarity. Nature 422:766-774

Niederkofler V, Salie R, Sigrist M, Arber S (2004) Repulsive guidance molecule (RGM) gene function is required for neural tube closure but not retinal topography in the mouse visual system. J Neurosci 24:808-818.

Nieuwkoop P, Faber J (1994) Normal table of Xenopus laevis (daudin): Amsterdam: Elsevier North-Holland Biomedical.

Papan C, Campos-Ortega JA (1994) On the formation of the neural keel and neural tube in the zebrafish Danio (brachydanio) rerio. Roux's Arch Dev Biol 203:178-186.

Park KW, Crouse D, Lee M, Karnik SK, Sorensen LK, Murphy KJ, Kuo CJ, Li DY (2004) The axonal attractant netrin-1 is an angiogenic factor. Proc Natl Acad Sci U S A 101:16210-16215.

Pilot F, Lecuit T (2005) Compartmentalized morphogenesis in epithelia: from cell to tissue shape. Dev Dyn 232:685-694.

Rajagopalan S, Deitinghoff L, Davis D, Conrad S, Skutella T, Chedotal A, Mueller BK, Strittmatter SM (2004) Neogenin mediates the action of repulsive guidance molecule. Nat Cell Biol 6:756-762.

Ruiz i Altaba A (1994) Pattern formation in the vertebrate neural plate. Trends Neurosci 17:233-243.

Schmitz B, Papan C, Campos-Ortega JA (1993) Neurulation in the anterior trunk region of the zebrafish Brachydanio rerio. Roux's Arch Dev Biol 202:250-259.
Schroeder TE (1970) Neurulation in Xenopus laevis. An analysis and model based upon light and electron microscopy J Embryol Exp Morphol 23:427-462.

Schroeder TE (1971) Mechanisms of morphogenesis: the embryonic neural tube. Int J Neurosci 2:183-197.

Srinivasan K, Strickland P, Valdes A, Shin GC, Hinck L (2003) Netrin-1/ neogenin interaction stabilizes multipotent progenitor cap cells during mammary gland morphogenesis. Dev Cell 4:371-382.

Vielmetter J, Kayyem JF, Roman JM, Dreyer WJ (1994) Neogenin, an avian cell surface protein expressed during terminal neuronal differentiation, is closely related to the human tumor suppressor molecule Deleted in Colorectal Cancer. J Cell Biol 127:2009-2020.

Wallingford JB (2005) Neural tube closure and neural tube defects: studies in animal models reveal known knowns and known unknowns. Am J Med Genet C Semin Med Genet 135:59-68.

Wallingford JB, Harland RM (2002) Neural tube closure requires dishevelled-dependent convergent extension of the midline. Development 129:5815-5825.

Wilson NH, Key B (2006) Neogenin interacts with RGMa and netrin-1 to guide axons within the embryonic vertebrate forebrain. Dev Biol 296:485-498.

Ybot-Gonzalez P, Cogram P, Gerrelli D, Copp AJ (2002) Sonic hedgehog and the molecular regulation of mouse neural tube closure. Development 129:2507-2517.

Ybot-Gonzalez P, Gaston-Massuet C, Girdler G, Klingensmith J, Arkell R, Greene ND, Copp AJ (2007) Neural plate morphogenesis during mouse neurulation is regulated by antagonism of Bmp signaling. Development 134:3203-3211. 